Mens

revue d'histoire intellectuelle de l'Amérique française

\title{
"La vocation de la race française en Amérique ", de Monseigneur Louis-Adolphe Pâquet
}

\section{Dominique Foisy-Geoffroy}

Volume 3, numéro 1, automne 2002

URI : https://id.erudit.org/iderudit/1024619ar

DOI : https://doi.org/10.7202/1024619ar

Aller au sommaire du numéro

Éditeur(s)

Centre de recherche en civilisation canadienne-française

ISSN

1492-8647 (imprimé)

1927-9299 (numérique)

Découvrir la revue

Citer ce document

Foisy-Geoffroy, D. (2002). « La vocation de la race française en Amérique ", de Monseigneur Louis-Adolphe Pâquet. Mens, 3(1), 61-95.

https://doi.org/10.7202/1024619ar d'utilisation que vous pouvez consulter en ligne. 


\title{
Documents
}

\section{« LA VOCATION DE LA RACE FRANÇAISE EN AMÉRIQUE », DE MONSEIGNEUR LOUIS-ADOLPHE PÂQUET ${ }^{1}$}

\author{
Édition commentée par \\ Dominique Foisy-Geoffroy \\ Département d'histoire \\ Université Laval
}

Rarement discours a-t-il eu si grande résonance au Canada français que celui qui fut prononcé à Québec en 1902 par $\mathrm{M}^{\mathrm{gr}}$ Louis-Adolphe Pâquet à l'occasion de la Saint-JeanBaptiste. Porté aux nues de façon aussi brillante qu'élogieuse par le chanoine Émile Chartier, qui en a fait en 1925 le Bréviaire du patriote canadien-français ${ }^{2}$, il fut cependant jugé le plus souvent avec dureté et un soupçon de condescendance par les historiens contemporains qui l'élevèrent pour leur part au rang de symbole de l'aliénation des Canadiens français. Qu'on ait fait de ce discours le signe éclatant de l'idéalisme de la pensée dite " clérico-nationaliste » et du décalage entre cette pensée et la réalité socio-économique québécoise, ou qu'on se soit livré à une analyse de type séguiniste ${ }^{3}$, si on peut dire, qui fait de ce discours un cas type de " déformation idéologique » produit par une pratique, par un " agir » diminués, on aboutit à une condamnation sans appel ${ }^{4}$. Cette condamnation, il faut le dire, se base habituellement sur un extrait du texte, très célèbre, qu'on suppose représentatif du discours : 
"Notre mission est moins de manier des capitaux que de remuer des idées ; elle consiste moins à allumer le feu des usines qu'à entretenir et à faire rayonner au loin le foyer lumineux de la religion et de la pensée. »

Au fond, que reproche-t-on à Pâquet ? De se désintéresser de l'économique, de la vie matérielle et, sans doute, de faire fi de l'aspiration des gens au bonheur terrestre. Peut-être aussi lui tient-on rigueur de ne pas avoir compris la corrélation entre la prospérité économique et l'épanouissement culturel, et même spirituel, des sociétés et des hommes. Il s'agit en somme d'une critique à saveur matérialiste adressée à une pensée qu'on juge excessivement antimatérialiste. Et pourtant, qu'en est-il ? Une relecture de ce texte nous a amené à formuler une interprétation différente.

\section{Qui est $\mathrm{M}^{\mathrm{gr}}$ Pâquet ?}

Louis-Adolphe Pâquet naît dans une famille d'agriculteurs à Saint-Nicolas, dans la grande région de Québec, le 4 août 1859. Après des études au Petit Séminaire de Québec entre 1872 et 1878, il part à Rome poursuivre des études théologiques et philosophiques (1879-1883), à la suite desquelles il obtient un doctorat en théologie de la Propagande soutenu devant, entre autres, le pape Léon XIII. En cette même année 1883, il est ordonné prêtre et revient à Québec. Dès son retour, il devient professeur à la Faculté de théologie de l'Université Laval, poste qu'il occupera jusqu'à sa mort. Il amorce ainsi une brillante carrière universitaire, marquée notamment par sa nomination au Conseil de Direction de l'université (1898-1938), par son accession au titre de doyen de la Faculté de théologie (1904-1938), et par sa nomination à la direction de l'École supérieure de philosophie (1926), la future Faculté de philosophie de l'Université Laval. Pâquet, en outre, est un homme engagé dans le siècle, qui se manifeste entre 
autres à l'occasion du débat sur la question scolaire au Manitoba (décennie 1890-1900), du litige entourant le Règlement XVII, promulgué par le ministère de l'Éducation de l'Ontario (années 1910-1930) ainsi que de la crise de la conscription (1917).

Sur le plan intellectuel, Pâquet est très proche des idées de saint Thomas d'Aquin, remises à l'honneur par Léon XIII, et s'érige en fervent défenseur de la Doctrine sociale de l'Église. Ses trois productions intellectuelles les plus marquantes témoignent de cet engagement. Il s'agit de Disputationes theologicae, seu, commentaria in Summam theologicam D. Thomae, un commentaire en six volumes de la Somme théologique de Saint Thomas d'Aquin, de Droit public de l'Église ${ }^{6}$, où il traite entre autres des rapports entre l'Église et le pouvoir civil, et des Études et appréciations ${ }^{7}$ dans lesquelles il interprète différents thèmes (la vie sociale, la vie canadienne) à la lumière de la Doctrine sociale de l'Église et du thomisme.

Pâquet, qualifié de "French-Canadian ultramontanism's most articulate exponent ${ }^{8}$ ", de " chef de file de la pensée ecclésiastique au Québec de 1896 à $1930^{\circ}$ », est un intellectuel catholique, nationaliste et traditionaliste dont la pensée, très systématique, se caractérise par une grande fidélité aux enseignements de la Doctrine sociale de l'Église, à la papauté et aux principes thomistes, et est marquée d'un souci constant d'appliquer ces enseignements aux conditions du milieu canadien-français ${ }^{10}$. Bref, pour Pâquet, la théorie, les principes doivent déboucher sur l'action.

\section{"La vocation de la race française en Amérique "}

Comme nous l'avons dit, le texte le plus célèbre de LouisAdolphe Pâquet est sans aucun doute son discours intitulé La vocation de la race française en Amérique. C'est à ce texte que nous consacrons le reste de cette présentation. 
a) Le contexte

Cette fin de juin 1902 est une période d'intenses réjouissances à Québec. On y célèbre en effet à la fois la SaintJean-Baptiste (23 juin), le cinquantenaire de l'Université Laval (24 juin) et le soixantième anniversaire de la Société SaintJean-Baptiste de Québec. Au matin du 23 juin, une grande procession de la Saint-Jean se met en branle et parcourt les rues de la ville, pour s'arrêter - et ce n'est pas un hasard - à l'entrée de la terrasse Dufferin, au pied du Château Frontenac, là où se trouve la statue de Samuel de Champlain qui orne encore l'endroit de nos jours. On y tient alors une grandmesse en plein air, célébrée par l'archevêque de Québec, $\mathrm{M}^{\mathrm{gt}}$ Louis-Nazaire Bégin. Le discours de Pâquet est en fait le sermon qui fut prononcé durant cette messe ${ }^{11}$. On a peut-être tendance à l'oublier, ce texte est donc d'abord un discours patriotique. Comme tout discours prononcé dans ce genre de circonstances, il propose avant tout à son auditoire un idéal mobilisateur, dont la hauteur est à la mesure d'un potentiel national que l'orateur cherche à valoriser. Plus précisément, Pâquet cherche à faire réaliser aux Canadiens français l'ampleur de leur destinée, qui est leur "vocation apostolique». Ils ont la fibre des forgeurs de civilisation, leur dit-il, et cette vocation est la plus belle qui puisse être pour un peuple. Par conséquent ils n'ont pas à rougir de ce qu'ils sont, bien au contraire. Cet idéal, cependant, ne se comprend dans son sens plein qu'à la lumière de ce qu'on pourrait appeler la "vision du monde » de Pâquet, une vision traditionaliste. C'est ainsi que le discours de Pâquet, outre son caractère de plaidoyer patriotique solennel et émotif, se présente sous un autre jour, soit comme un exposé systématique de la plupart des thèmes chers au traditionalisme canadien-français. C'est certainement, à notre avis, en raison de ce double caractère que le chanoine Chartier en a fait le "Bréviaire du patriote canadien-français », 
expression à la fois synthétique, systématique et mobilisatrice, exemplaire de l'idéal canadien-français qui animait les traditionalistes d'ici.

\section{b) Mise en scène}

D'entrée de jeu, l'auteur présente un tableau édifiant qui, à lui seul, est très révélateur de l'atmosphère du texte. Aux origines de la colonie, une messe est dite au pied du Cap Diamant, à Québec, où le noyau de la population se réunit. Près de 300 ans plus tard, la nation " issue de cette semence féconde " s'assemble de nouveau, " non plus au pied de la falaise, mais sur ses hauteurs ", pour réactualiser un moment qui est symboliquement élevé au rang d'acte fondateur de la nation. Entre les deux, un immense travail de civilisation, d'humanisation du milieu, s'est opéré, travail qui constitue la substance même de l'histoire et qui a fait une nation de la collectivité d'hommes et de femmes qui en sont les ouvriers. Cependant, toute cette évolution, que le passage du pied de la falaise à son sommet illustre autant géographiquement que symboliquement, procède d'un esprit qui, lui, est resté constant au travers des siècles. C'est cette constance qu'exprime l'image de la réactualisation d'une messe célébrée aux origines de la colonie, en même temps qu'elle révèle, de façon implicite, quelle est l'essence de la nation, de la tradition canadienne-française : les grandes valeurs, les grands principes hérités de la vénérable culture chrétienne et française prérévolutionnaire.

Il faut noter en passant un fait extrêmement intéressant. À la fin du texte, Pâquet reviendra sur ce tableau d'introduction en le décrivant comme un "pacte social ", pacte qui, doit-on le préciser, n'a rien de rousseauiste dans son entendement : 
L'Église et l'État, le clergé et les citoyens, toutes les sociétés, toutes les classes, tous les ordres, toutes les professions, se sont donné la main pour venir au pied de l'autel, en face de Celui qui fait et défait les empires, renouveler l'alliance étroite conclue non loin d'ici, à la naissance même de cette ville, entre la patrie et Dieu.

Cette référence à l'un des thèmes récurrents de la philosophie politique moderne des $\mathrm{XVII}^{\mathrm{e}}$ et $\mathrm{XVIII}^{\mathrm{e}}$ siècles pour désigner la nature du lien qui unit chacun aux autres et à Dieu dans la conception traditionaliste, référence présentée, de surcroît, dans des termes qui évoquent l'alliance de Dieu avec le peuple hébreu, n'est pas qu'un clin d'œil, mais une réappropriation. Elle est une réponse aux conceptions de la philosophie politique moderne, et sans doute tout particulièrement au libéralisme. Contre l'image d'une société divisée en deux sphères, soit d'une part une société politique formée de citoyens désincarnés dont on postule la liberté et l'égalité et d'autre part une société civile dont le marché constitue le principal mécanisme régulateur, Pâquet présente la société comme un tout organique où chacun tient une place déterminée et est lié à l'ensemble par une multitude de liens (famille, nation, classe sociale, etc.), un tout organique sur lequel Dieu, source de toute autorité, veille. Il n'en demeure pas moins que la nation entière est ainsi, implicitement, identifiée comme dépositaire de cette autorité. La conception de Pâquet intègre donc, à sa façon, les formes modernes de légitimité du pouvoir. Pâquet n'invente rien cependant en récupérant ainsi le concept de " pacte » : Edmund Burke ${ }^{12}$, notamment, et sans doute de nombreux autres penseurs aux positions conservatrices, ont aussi tenu ce genre de propos.

Pour Pâquet, cela dit, cette " grand-messe nationale » est, outre la cause de légitimes réjouissances, l'occasion de réaffirmer à ses compatriotes «ce que nous avons été, ce que 
nous sommes, ce que nous devons et voulons être ", expression qui révèle le traditionalisme de l'auteur, soit une conscience poussée de la continuité historique et l'idée de destin qui s'y profile : le sens de l'avenir d'un peuple est à chercher dans ses origines. Cette intention se résout en un exposé méthodique sur la "vocation de la race française en Amérique ».

\section{c) La vocation des nations}

Le corps du texte est divisé en deux grandes sections. Dans la première, l'auteur affirme que les peuples ont une vocation. Puis, dans la seconde, il prouve par le recours à l'histoire que la vocation du peuple canadien-français est essentiellement sacerdotale.

Que les nations aient une vocation, cela est inscrit, pour Pâquet, dans l'ordre des choses et du monde. Dans son esprit, cet ordre est en fait le fruit d'une Idée, de la volonté divine, mise en œuvre, du moins en ce qui concerne les choses humaines, par le génie des hommes. Cet ordre du monde est tel que chaque nation, comme chaque individu d'ailleurs, y occupe une place déterminée et contribue, à sa façon propre, à l'équilibre et au bon fonctionnement de l'ensemble. Bref, la nation a un rôle bien précis à jouer, et ce rôle est sa vocation, sa raison d'être. Ce qu'on observe, et cela est de toute évidence un aspect fondamental de la pensée de $\mathrm{M}^{\mathrm{rr}}$ Pâquet, c'est que cette vision du monde est fondamentalement spiritualiste et nationaliste. Il développera d'ailleurs clairement cette idée un peu plus loin dans le texte :

Car on a beau dire, mes Frères, c'est la science qui mène le monde. Cachées sous le voile des sens ou derrière l'épais rideau de la matière, les idées abstraites demeurent, il est vrai, invisibles ; mais semblables à cette force motrice que personne ne voit et qui distribue partout avec une si merveilleuse précision la lu- 
mière et le mouvement, ce sont elles qui inspirent tous les conseils, qui déterminent toutes les résolutions, qui mettent en branle toutes les énergies.

Déterministe, cette conception ? Sans doute y a-t-il de forts relents de providentialisme dans ce texte. On sent que dans l'esprit de Pâquet, l'histoire a un sens et que la main de Dieu s'y profile. Pourtant, et malgré cela, elle est certainement beaucoup moins déterministe qu'on pourrait le penser de prime abord. C'est que la volonté divine, en ce qui concerne les choses humaines, est mise en œuvre par l'homme après que celui-ci l'a reconnue et a librement et consciemment résolu de s'y conformer. Un individu pourrait donc décider, pour toutes sortes de (mauvaises) raisons, de ne pas accorder sa vie avec cet ordre du monde qui le dépasse, mais alors il devra assumer les conséquences de ce choix. Cela s'applique de la même façon aux nations, qui, elles, risquent la destruction :

Une nation peut déchoir des hauteurs de sa destinée. Cela n'accuse ni impuissance ni imprévoyance de la part de Dieu ; la faute en est aux nations elles-mêmes qui, perdant de vue leur mission, abusent obstinément de leur liberté et courent follement vers l'abîme.

En somme, dans la perspective traditionaliste de Pâquet, il existe indéniablement une part de liberté chez l'homme. Cependant, l'usage de cette liberté, pour porter tous ses fruits, doit s'inscrire dans le cadre d'une recherche de l'ordre (constitué de normes générales : le beau, le vrai, le bien) censé assurer le bonheur de l'homme, considéré avant tout comme un être culturel et spirituel.

\section{d) Une vocation prouvée par l'histoire}

Pour Pâquet, il ne fait aucun doute que la « race française en Amérique » est investie d'une mission sacerdotale, la 
plus haute qui puisse être : « Nous ne sommes pas seulement une race civilisée, nous sommes des pionniers de la civilisation; nous ne sommes pas seulement un peuple religieux, nous sommes des messagers de l'idée religieuse. "Suit le célèbre passage que nous avons cité en introduction, qui est à peu près tout ce qu'on a retenu de ce texte décidément beaucoup plus riche qu'on a pu le croire.

De l'avis de l'auteur, c'est l'histoire de l'Amérique française qui fournit les démonstrations les plus probantes de cette vocation sacerdotale. Dans un premier temps, il fait valoir que cette vocation est inscrite dans l'origine même de la nation :

Quelle est donc la nation mère à laquelle nous devons l'existence ? quel a été son rôle, son influence intellectuelle et sociale ? Déjà vos cœurs émus ont désigné la France ; et, en nommant cette patrie de vos âmes, ils évoquent, il ressuscitent toute l'histoire du christianisme.

Pâquet fait ainsi référence à l'essence catholique et française de la nation, qu'elle tire de ses origines mêmes. Dans un second temps, il montre que son histoire est elle-même marquée du sceau de cette vocation, des premières heures de la colonie à l'époque contemporaine. En effet, les débuts de la Nouvelle-France ont selon lui été marqués de façon dominante par l'action missionnaire des zélateurs du catholicisme :

Qui, mes Frères, ne reconnaitrait cette mission, en voyant les plus hauts personnages dont notre histoire s'honore, faire de l'extension du royaume de Jésus-Christ le but premier de leurs entreprises et marquer, pour ainsi dire, chacune de leurs actions d'un cachet religieux ?

Et Pâquet d'évoquer l'action religieuse des Cartier, Champlain, Maisonneuve, Marie de l'Incarnation, $\mathrm{M}^{\mathrm{gr}}$ de Laval et consorts, et de souligner avec insistance la bienveillance toute chrétienne des Français à l'endroit des Amérindiens. 
Évidemment, il s'agit là d'une interprétation orientée de ce qu'était la Nouvelle-France, les motifs de sa fondation et de sa perpétuation durant un siècle et demi étant beaucoup plus complexes. Entre les objectifs économiques et commerciaux, politiques, militaires, il est sans doute téméraire d'affirmer que le motif religieux fut dominant. Pourtant, il n'en était pas moins présent. Des missionnaires récollets et jésuites aux fondateurs de Ville-Marie, en passant par les communautés de religieuses éducatrices et hospitalières, les apôtres du catholicisme furent nombreux dans la colonie, et leur grande foi informa certainement le caractère de cette colonisation. En ce sens, l'interprétation de $\mathrm{M}^{\mathrm{gr}}$ Pâquet est plus incomplète et simplificatrice qu'erronée.

Il importe ensuite à l'auteur de déterminer si la nation a su préserver les caractères qui en ont fait un peuple élu à une vocation sacerdotale, ou, en d'autres mots, si elle fut fidèle à son essence au cours des siècles et jusqu'à l'orée du $\mathrm{XX}^{\mathrm{e}}$, au rôle que sa place dans l'ordre du monde lui confère. À cette question, l'auteur répond fermement par l'affirmative en invoquant un certain nombre de signes typiques de la survivance et même de la progression du fait catholique et français en Amérique : l'extension du domaine habité dans le Québec par la colonisation (menée sous la houlette de valeureux prêtres-colonisateurs), la croissance de l'Université Laval (foyer de pensée), la survivance des groupes d'émigrés canadiensfrançais dans les autres provinces canadiennes et aux ÉtatsUnis ainsi que les progrès de l'Acadie. En somme la boucle est bouclée : l'Amérique française a su préserver son être et rester fidèle à sa vocation au cours des siècles, comme le montre son histoire.

D'ailleurs, ce recours à l'histoire est à notre avis assez significatif et prend une valeur bien particulière dans la perspective traditionaliste qu'adopte Pâquet. En effet, la connais- 
sance de l'histoire se présente ainsi comme le moyen privilégié pour l'individu de développer le sens de la solidarité avec les ancêtres et de la continuité avec les principes qui ont présidé à l'humanisation du milieu. Cela n'exclut pas tout rapport critique avec le passé, mais marque simplement une révérence devant un héritage, un " ordre » qui est le fruit de plusieurs siècles de pensée et d'action, dont la seule durée témoigne en bonne partie de la juste inspiration et invite à une grande prudence dans toute entreprise réformatrice. Inspiré par sa connaissance de l'histoire, le citoyen est invité à puiser dans la tradition à la fois des règles de vie et des façons d'exercer ses responsabilités civiques qui, parce qu'elles sont conformes aux principes séculaires ordonnateurs de la nation, sont garants de l'épanouissement le plus complet de l'homme. L'argument historique est donc coiffé d'une auréole qui affirme son autorité.

\section{e) Chemins de l'avenir}

Une fois posée la vocation sacerdotale de la « race française en Amérique » et démontrée la fidélité de la nation à cette vocation, il reste à établir les moyens de préserver cette fidélité dans l'avenir pour assurer à l'Amérique française des lendemains qui chantent. C'est l'objet de la deuxième section du texte de Pâquet.

Au total, on peut identifier dans le texte six conditions susceptibles de permettre la survie et l'épanouissement de la nation. Premièrement, un peuple doit « rester lui-même », c'està-dire être fidèle à la place qu'il occupe dans l'ordre du monde, aux grands principes qui ont présidé à l'action du milieu par l'homme et qui a produit la nation. Concrètement, " un peuple ne reste lui-même que par la liberté de sa vie, l'usage de sa langue, la culture de son génie ». Ce qu'il importe de souligner, c'est le flou relatif des trois paragraphes consacrés à 
cette condition de vie primordiale, sans doute dû au fait que la réalisation de cette exigence implique de s'interroger sur la structure politique à l'intérieur de laquelle évolue la nation, ce dont, en principe, doit se garder un membre de la hiérarchie catholique. La Doctrine sociale de l'Église est assez claire là-dessus. Pâquet doit ainsi jouer de prudence : «Il ne m'appartient pas ici de discuter l'avenir politique de mon pays. Mais... »

On comprendra mieux tout ceci en se questionnant d'abord sur ce qu'est, plus précisément, "rester soi-même » pour un peuple. Pour Pâquet, il nous semble que cela veut dire avoir la liberté de pratiquer sa religion, de parler sa langue et, plus généralement, d'humaniser le milieu (notamment faire évoluer les cadres de la vie collective - politiques, économiques, sociaux) en continuité avec l'action des ancêtres et les grands principes qui ont informé ce travail civilisateur depuis les origines. Il écrira par exemple ce qui suit en conclusion de son texte :

Cette charte [où sont consignés les termes du " pacte » dont nous avons parlé précédemment, et où est fixée la vocation de la « race française en Amérique »], où sont inscrits tous les droits, où sont reconnues toutes les saintes libertés, qu'elle soit promulguée partout, sur les portes de nos cités, sur les murs de nos temples, dans l'enceinte de nos parlements et de nos édifices publics ! Qu'elle dirige nos législateurs, qu'elle éclaire nos magistrats, qu'elle inspire tous nos écrivains! Qu'elle soit la loi de la famille, la loi de l'école, la loi de l'atelier, la loi de l'hôpital! Qu'elle gouverne, en un mot, la société canadienne tout entière!

Cela implique que les institutions politiques ménagent une autonomie suffisante à la nation pour qu'elle puisse jouir du « libre jeu de son organisme et de sa vie ». Dans la perspective d'un Canada binational qui est certainement celle de 
Pâquet, les institutions politiques canadiennes, tant fédérales que provinciales, devraient donc permettre aux deux principaux groupes nationaux qui constituent le pays de s'épanouir librement. Pour ce qui est du Canada français, c'est à juste titre à notre avis qu'Émile Chartier rappelle que l'invitation faite par Pâquet à son peuple " de garder dans une humble sphère le libre jeu de son organisme et de sa vie [plutôt] que de graviter dans l'orbite de vastes systèmes planétaires " s'inscrit dans le débat, très vif à l'époque, qui divise les tenants du nationalisme canadien des partisans d'une intégration accrue du Canada à la vie de l'Empire britannique, les impérialistes. Il semble évident aux yeux de Pâquet que l'intégration poussée du Canada français à l'Empire, ensemble à la fois si vaste, si étranger à l'âme canadienne-française et de surcroît sans doute peu intéressé à son épanouissement, ne servirait qu'à le perdre.

Deuxièmement, un peuple doit s'accrocher à sa langue comme à un joyau de civilisation, et à plus forte raison s'il s'agit de la langue française, qui a « l'honneur de porter comme dans un écrin le trésor de la pensée humaine enrichi de toutes les traditions des grands siècles catholiques ». Ce qui apparaît assez clairement, c'est le lien très fort qui unit la langue à la pensée, que non seulement elle communique, mais, davantage encore, qu'elle incarne. Ainsi, elle devient en quelque sorte dans la perspective de Pâquet l'agent civilisateur par excellence.

Troisièmement, la " race française d'Amérique » doit garder à l'esprit la primauté des aspirations culturelles et spirituelles de l'homme - d'où l'importance de ce lieu d'étude et de pensée qu'est l'Université Laval, - mais, et cela est capital pour notre propos, sans négliger les liens qui unissent la vie spirituelle aux aspects matériels de la vie humaine, et notamment à l'économique. C'est en suivant le fil de cette argu- 
mentation qu'on retrace, dans le détail et les nuances, la pensée de Pâquet concernant le Canada français et la vie économique :

À Dieu ne plaise, mes Frères, que je méprise ces bienfaits naturels de la Providence [les richesses matérielles], et que j’aille jusqu'à prêcher à mes concitoyens un renoncement fatal aux intérêts économiques dont ils ont un si vif souci. La richesse n'est interdite à aucun peuple ni à aucune race ; elle est même la récompense d'initiatives fécondes, d'efforts intelligents et de travaux persévérants.

La poursuite des richesses matérielles est donc légitime. Cependant, Pâquet sert immédiatement une mise en garde à ceux qui seraient tentés d'en faire un absolu, mise en garde qui a par ailleurs l'effet un peu paradoxal d'ajouter à la valeur de la vie économique - d'une vie économique bien entendue - en faisant d'une occupation légitime une nécessité :

Mais prenons garde ; n'allons pas faire de ce qui n'est qu'un moyen le but même de notre action sociale. [...] Usons des biens matériels, non pour eux-mêmes, mais pour les biens plus précieux qu'ils peuvent nous assurer; usons de la richesse, non pour multiplier les vils plaisirs des sens, mais pour favoriser les plaisirs plus nobles, plus élevés de l'âme ; usons du progrès, non pour nous étioler dans le béotisme qu'engendre trop souvent l'opulence, mais pour donner à nos esprits des ailes plus larges et à nos cœurs un plus vigoureux élan.

La poursuite des richesses matérielles est donc légitime et même nécessaire pourvu qu'elle soit comprise comme un moyen facilitant l'accomplissement des fins spirituelles et culturelles de l'humanité, les plus hautes qui soient. En somme, le texte de Pâquet n'est pas tant une dénonciation de l'activité économique qu'un ferme rappel de sa place, subordonnée mais néanmoins importante, dans la hiérarchie des 
aspects de la vie humaine, ainsi que des conditions qui font de son exercice un enrichissement pour l'humanité, et non une aliénation. Il nous semble donc qu'Yvan Lamonde se méprend lorsqu'il écrit que Pâquet a "nuancé " sa position durant la crise des années 1930 sur les rapports du Canada français à la vie économique, en affirmant l'importance « d'imprimer sur le terrain économique le sceau de notre esprit et de nos croyances $^{13}$ ». Cela était à l'œuvre, et de façon assez explicite, dès 1902. Ainsi la position de Pâquet durant la crise ne paraît accuser aucun changement fondamental par rapport à ses positions antérieures.

Finalement, les quatrième, cinquième et sixième conditions, rapidement présentées, sont la nécessité de faire déboucher la pensée sur l'action, la solidarité nationale (qui doit être poussée « aussi loin que le permettent [les] devoirs de loyauté politique »), ainsi que la fidélité à l'Église, incontournable pour toute nation chrétienne.

\section{$* * *$}

Que conclure au terme de cette analyse ? S'il nous faut invalider la thèse d'une dénonciation de l'activité économique par Pâquet, certains passages du texte ne laissent toutefois pas d'évoquer une sorte de complexe du Canadien français face à sa position défavorable sur le plan économique. Il semble parfois que l'auteur ait renversé intellectuellement ce sentiment d'infériorité d'une manière qui sonne faux et ne convainc pas : l'esprit canadien-français est appelé à flotter dans l'éther de la haute pensée et non pas à se complaire dans la poursuite de viles fins matérielles... «Pendant que nos rivaux revendiqueront, sans doute dans des luttes courtoises, l'hégémonie de l'industrie et de la finance, nous, fidèles à notre vocation première, nous ambitionnerons avant tout l'honneur de la doctrine et les palmes de l'apostolat. » Pourtant, si cette 
explication semble avoir quelque fondement, notre analyse a montré qu'elle était bien secondaire par rapport à ce qui nous semble être le véritable motif du texte de Pâquet : un idéal traditionaliste soucieux de porter l'homme à ses sommets et qui ne néglige pas la part, subordonnée mais décisive, des aspects temporels de la vie humaine - y compris de l'économique - dans la réalisation des plus hautes aspirations humaines. Bien plus que quelque aliénation du Canadien français, c'est donc avant tout une vision du monde, complète, nuancée, exigeante, et un véritable projet de civilisation, qui sont exposés ici. 


\section{LA VOCATION DE LA RACE FRANÇAISE EN AMÉRIQUE}

\section{par $M^{g r}$ Louis-Adolphe Pâquet 23 juin $1902^{14}$}

Le vingt-cinq juin seize cent quinze, à quelques pas d'ici, sur cette pointe de terre qui du pied de la falaise où nous sommes s'avance dans l'eau profonde de notre grand fleuve, se déroulait une scène jusque-là inconnue. À l'ombre de la forêt séculaire, dans une chapelle hâtivement construite, en présence de quelques Français et de leur chef, Samuel de Champlain, un humble fils de saint François ${ }^{15}$, tourné vers un modeste autel, faisait descendre sur cette table rustique le Fils éternel de Dieu, et lui consacrait par l'acte le plus saint de notre religion les premiers fondements d'une ville et le berceau d'un peuple.

Ce peuple, depuis lors, a grandi. Cette ville a prospéré, et voici qu'à une distance d'à peu près trois siècles la nation, issue de cette semence féconde, s'assemble, non plus au pied de la falaise, mais sur ses hauteurs, pour renouveler son acte de consécration religieuse et retremper sa vie dans le sang de l'Agneau divin.

Quelles transformations et quels contrastes ! Tout autour, malgré l'immuabilité des grandes lignes qui forment le cadre du tableau, la nature a reçu l'empreinte de l'esprit et de la main de l'homme, le désert s'est animé, les solitudes se sont peuplées. Plus près de nous, au lieu de tentes mobiles où s'abritait la barbarie, l'œil contemple de massifs châteaux et d'artistiques édifices ; des tours, des flèches altières ont remplacé la cime des pins ; toute une civilisation déjà adulte a surgi ; et le fondateur de Québec, du haut de ce monument que lui élevait naguère la reconnaissance publique, fier de son 
œuvre, plus fier encore des progrès merveilleux qui en ont marqué la durée, peut plonger dans l'avenir un regard plein d'espoir et saluer avec confiance l'aube blanchissante de jours nouveaux et de destinées de plus en plus glorieuses.

Mes Frères, c'est pour envisager ce même avenir que nous sommes ici ce matin. Le cor résonnant de nos fêtes patriotiques a retenti, et des quatre coins de la Province, des extrémités du pays, je pourrais presque dire, de tous les points de l'Amérique où la race française a planté son drapeau, vous êtes accourus en foule, la tête haute, le cœur vibrant. On ne pouvait répondre à l'appel avec plus d'unanimité ni avec plus d'enthousiasme.

Aussi bien, le moment est solennel. Et sous ces airs de fête et à travers cet éclat de nos communes réjouissances, je vois des esprits qui s'inquiètent, des regards qui interrogent, des fronts sur lesquels se traduisent de soucieuses pensées ; j'entends d'une part, des clameurs vagues et confuses, et, de l'autre, comme l'écho d'émotions contenues et de secrets frémissements passant dans l'âme de la nation. Que signifie cela?

C'est que, mes Frères, dans notre marche historique, nous sommes parvenus à une de ces époques où les peuples prennent conscience d'eux-mêmes, de leur vitalité et de leur force. C'est que, en assistant aux manifestations grandioses provoquées par d'heureux anniversaires de notre vie intellectuelle et sociale, nous sommes en même temps et plus spécialement peut-être conviés à de véritables assises nationales. C'est que, dans ces assises, il s'agit pour nous d'étudier et d'approfondir le problème de nos destinées et de proclamer une fois de plus, sans forfanterie comme sans faiblesse, prudemment, sagement, ce que nous avons été, ce que nous sommes, ce que nous devons et voulons être.

Voilà pourquoi je vous citais tout à l'heure ces paroles de nos lettres sacrées: Populum istum formavi mibi; laudem meam 
narrabit ${ }^{16}$. C'est moi qui ai formé ce peuple, et je l'ai établi pour qu'il publie mes louanges. Dans ce langage, en effet, d'une si haute signification, et à travers ces accents inspirés, j'aperçois des indices de la noble mission confiée à notre nationalité ; je crois découvrir, à cette lumière, la sublime vocation de la race française en Amérique.

\section{I}

Y-a-t-il donc, mes Frères, une vocation pour les peuples?

Ceux-là seuls peuvent en douter qui écartent des événements de ce monde la main de la Providence et abandonnent les hommes et les choses à une aveugle fatalité. Quant à nous qui croyons en Dieu, en un Dieu sage, bon et puissant, nous savons comment cette sagesse, cette bonté et cette puissance se révèlent dans le gouvernement des nations ; comment l'Auteur de tout être a créé des races diverses, avec des goûts et des aptitudes variés, et comment aussi il a assigné à chacune de ces races, dans la hiérarchie des sociétés et des empires, un rôle propre et distinct. Une nation sans doute peut déchoir des hauteurs de sa destinée. Cela n'accuse ni impuissance ni imprévoyance de la part de Dieu ; la faute en est aux nations elles-mêmes qui, perdant de vue leur mission, abusent obstinément de leur liberté et courent follement vers l'abîme.

Je vais plus loin, et j'ose affirmer que non seulement il existe une vocation pour les peuples, mais qu'en outre quelques-uns d'entre eux ont l'honneur d'être appelés à une sorte de sacerdoce. Ouvrez la Bible, mes Frères, parcourez-en les pages si touchantes, si débordantes de l'esprit divin, depuis Abraham jusqu'à Moïse, depuis Moïse jusqu'à David, depuis David jusqu'au Messie figuré par les patriarches, annoncé par les prophètes et sorti comme une fleur de la tige judaïque, et 
dites-moi si le peuple hébreu, malgré ses hontes, malgré ses défaillances, malgré ses infidélités, n'a pas rempli sur la terre une mission sacerdotale.

Il en est de même sous la loi nouvelle. Tous les peuples sont appelés à la vraie religion, mais tous n'ont pas reçu une mission religieuse. L'histoire tant ancienne que moderne le démontre : il y a des peuples voués à la glèbe, il y a des peuples industriels, des peuples marchands, des peuples conquérants, il y a des peuples versés dans les arts et les sciences, il y a aussi des peuples apôtres. Ah! reconnaissez-les à leur génie rayonnant et à leur âme généreuse : ce sont eux qui, sous la conduite de l'Église, ont accompli l'œuvre et répandu les bienfaits de la civilisation chrétienne; qui ont mis la main à tout ce que nous voyons de beau, de grand, de divin dans le monde ; qui par la plume, ou de la pointe de l'épée, ont buriné le nom de Dieu dans l'histoire ; qui ont gardé comme un trésor, vivant et impérissable, le culte du vrai et du bien. Ce sont ceux que préoccupent, que passionnent instinctivement toutes les nobles causes ; qu'on voit frémir d'indignation au spectacle du faible opprimé ; qu'on voit se dévouer, sous les formes les plus diverses, au triomphe de la vérité, de la charité, de la justice, du droit, de la liberté. Ce sont ceux, en un mot, qui ont mérité et méritent encore l'appellation glorieuse de champions du Christ et de soldats de la Providence.

Or, mes frères, - pourquoi hésiterais-je à le dire ? - ce sacerdoce social, réservé aux peuples d'élite, nous avons le privilège d'en être investis ; cette vocation religieuse et civilisatrice, c'est, je n'en puis douter, la vocation propre, la vocation spéciale de la race française en Amérique. Oui, sachonsle bien, nous ne sommes pas seulement une race civilisée, nous sommes des pionniers de la civilisation; nous ne sommes pas seulement un peuple religieux, nous sommes des messagers de l'idée religieuse ; nous ne sommes pas seulement 
des fils soumis de l'Église, nous sommes, nous devons être du nombre de ses zélateurs, de ses défenseurs et de ses apôtres. Notre mission est moins de manier des capitaux que de remuer des idées ; elle consiste moins à allumer le feu des usines qu'à entretenir et à faire rayonner au loin le foyer lumineux de la religion et de la pensée.

Est-il besoin que je produise des marques de cette vocation d'honneur? La tâche, mes Frères, est facile : ces marques, nous les portons au front, nous les portons sur les lèvres, nous les portons dans nos cœurs ${ }^{17}$ !

Pour juger de la nature d'une œuvre, d'une fondation quelconque, il suffit très souvent de reporter les yeux sur les débuts de cette œuvre, sur l'auteur de cette fondation. La vie d'un arbre est dans ses racines; l'avenir d'un peuple se manifeste dans ses origines. Quelle est donc la nation mère à laquelle nous devons l'existence ? quel a été son rôle, son influence intellectuelle et sociale ? Déjà vos cœurs émus ont désigné la France ; et, en nommant cette patrie de nos âmes, ils évoquent, ils ressuscitent toute l'histoire du christianisme. Le voilà le peuple apôtre par excellence, celui dont Léon XIII dans un document mémorable ${ }^{18}$ a pu dire : "La très noble nation française, par les grandes choses qu'elle a accomplies dans la paix et dans la guerre, s'est acquis envers l'Église catholique des mérites et des titres à une reconnaissance immortelle et à une gloire qui ne s'éteindra jamais. » Ces paroles si élogieuses provoqueront peut-être un sourire hésitant sur les lèvres de ceux qui ne considèrent que la France maçonnique et infidèle ${ }^{19}$. Mais, hâtons-nous de l'ajouter, dix ans, vingt ans, cent ans même de défections, surtout quand ces défections sont rachetées par l'héroïsme du sacrifice et le martyre de l'exil ${ }^{20}$, ne sauraient effacer treize siècles de foi généreuse et de dévouement sans égal à la cause du droit chrétien. 
Quand on descend d'une telle race, quand on compte parmi ses ancêtres des Clovis et des Charlemagne, des Louis IX et des Jeanne d'Arc, des Vincent de Paul et des Bossuet, n'eston pas justifiable de revendiquer un rôle à part et une mission supérieure $^{21}$ ? Par une heureuse et providentielle combinaison, nous sentons circuler dans nos veines du sang français et du sang chrétien. Le sang français seul s'altère et se corrompt vite, plus vite peut-être que tout autre ; mêlé au sang chrétien, il produit les héros, les semeurs de doctrines spirituelles et fécondes, les artisans glorieux des plus belles œuvres divines.

C'est ce qui explique les admirables sentiments de piété vive et de foi agissante dont furent animés les fondateurs de notre nationalité sur ce continent d'Amérique, et c'est dans ces sentiments mêmes que je trouve une autre preuve de notre mission civilisatrice et religieuse.

Qui, mes Frères, ne reconnaittrait cette mission, en voyant les plus hauts personnages dont notre histoire s'honore, faire de l'extension du royaume de Jésus-Christ le but premier de leurs entreprises et marquer, pour ainsi dire, chacune de leurs actions d'un cachet religieux ? Qui n'admettrait, qui n'admirerait cette vocation, en voyant, par exemple, un Jacques Cartier dérouler d'une main pieuse sur la tête de pauvres sauvages les pages salutaires de l'Évangile ${ }^{22}$; en voyant un Champlain ou un Maisonneuve mettre à la base de leurs établissements tout ce que la religion a de plus sacré ; en voyant encore une Marie de l'Incarnation et ses courageuses compagnes, à peine débarquées sur ces rives, se prosterner à terre ${ }^{23}$ et baiser avec transport cette patrie adoptive qu'elles devaient illustrer par de si hérö̈ques vertus? Est-ce donc par hasard que tant de saintes femmes, tant d'éminents chrétiens, tant de religieux dévoués se sont rencontrés dans une pensée commune et ont posé, comme à genoux, les premières pierres 
de notre édifice national ? Est-ce par hasard que ces pierres, préparées sous le regard de Dieu et par des mains si pures, ont été baignées, cimentées dans le sang des martyrs ${ }^{24}$ ? L'établissement de la race française dans ces contrées serait-il une méprise de l'histoire, et le flot qui nous déposa sur les bords du Saint-Laurent n'aurait-il apporté au rivage que d'informes débris, incapables de servir et d'accomplir les desseins du ciel dans une œuvre durable?

Non, mes Frères, et ce qui le prouve mieux encore que tout le reste, c'est l'influence croissante exercée autour d'elle par la France d'Amérique sur les progrès de la foi et de la vraie civilisation.

Chose digne de remarque, et qui jette une belle lumière sur la mission d'un peuple : chaque fois que nos ancêtres, dans leurs courses d'explorations et même dans leurs guerres, vinrent en contact avec les rudes enfants des bois, ce fut pour les civiliser plutôt que pour les dominer ; ce fut pour les convertir, et non pour les anéantir. Que n'ai-je le temps de rappeler les travaux de nos évêques, en particulier de l'immortel Laval, de nos prêtres, de nos missionnaires, de nos découvreurs, de tous nos apôtres ? C'est d'ici qu'est partie l'idée religieuse qui plane aujourd'hui sur une large portion de l'Amérique septentrionale. C'est ici qu'ont jailli ces sources de doctrine, de vertu, de dévouement, dont les ondes se sont propagées d'un océan à l'autre, et, devançant nos grandes routes de feu, ont porté aux races étrangères les trésors de christianisme dont la nôtre est dépositaire.

Et cette influence si étendue jadis, si puissante et si bienfaisante, menacerait-elle maintenant de décroitre ? Auraitelle du moins perdu, par le fait d'influences rivales, son caractère propre et ce cachet de spiritualisme qui l'a rendue si remarquable dans le passé ? Ah! demandez-le, mes Frères, aux vénérables prélats qui, par leur présence au milieu de nous, 
ajoutent à ces fêtes tant de lustre, et dont le sceptre, semblable à la verge de Moïse $\mathrm{e}^{25}$, a fait surgir comme par miracle de la bruyère inculte ou de l'épaisse forêt d'innombrables paroisses et de florissants diocèses. Demandez-le à cette Université, l'orgueil de notre patrie, dont l'enseignement projeté par un double foyer ${ }^{26}$ rayonne avec tant d'éclat, et qui après cinquante ans d'existence voit accourir vers elle, des diverses parties de ce continent, des milliers d'anciens élèves, sa joie et sa couronne. Demandez-le à tous ceux des nôtres que le souffle de l'émigration a dispersés loin de nous, soit dans d'autres provinces, soit sur le territoire de la vaste république américaine, et dont les groupes compacts, toujours catholiques, toujours français, resserrés autour de l'Église et de l'école paroissiale, émergent ça [sic] et là, comme de solides rochers au-dessus de la mer déferlante et houleuse. Demandez-le enfin à nos frères acadiens, chez qui le patriotisme, l'adhérence à la foi, l'attachement à la langue et l'indomptable ténacité, n’ont été égalés que par le malheur, et que Dieu récompense de tant de fidélité par une progression constante dans le nombre et dans l'influence ${ }^{27}$.

\section{Populum istum formavi mibi ; laudem meam narrabit. ${ }^{28}$}

C'est moi, dit le Seigneur, qui ai formé ce peuple ; je l'ai établi pour ma gloire, dans l'intérêt de la religion et pour le bien de mon Église ; je veux qu'il persévère dans sa noble mission, qu'il continue à publier mes louanges.

Oui, faire connaître Dieu, publier son nom, propager et défendre tout ce qui constitue le précieux patrimoine des traditions chrétiennes, telle est bien notre vocation. Nous en avons vu les marques certaines, indiscutables. Ce que la France d'Europe a été pour l'ancien monde, la France d'Amérique doit l'être pour ce monde nouveau. Mais dans l'état social où nous sommes, à quel prix, mes Frères, et par quels moyens 
remplirons-nous efficacement cette mission ? Quels sont les droits qu'elle comporte? quels sont les devoirs qu'elle impose ? Voilà ce dont il me reste à vous entretenir.

\section{II}

Pour exercer parmi les nations le rôle qui convient à sa nature et que la Providence lui a assigné, un peuple doit rester lui-même ; c'est une première et absolue condition, que rien ne saurait remplacer. Or, un peuple ne reste lui-même que par la liberté de sa vie, l'usage de sa langue, la culture de son génie. Il ne m'appartient pas de discuter ici l'avenir politique de mon pays. Mais ce que je tiens à dire, ce que je veux proclamer bien haut en présence de cette patriotique assemblée, c'est que le Canada français ne répondra aux desseins de Dieu et à sa sublime vocation que dans la mesure où il gardera sa vie propre, son caractère individuel, ses traditions vraiment nationales.

Et qu'est-ce donc que la vie d'un peuple? Vivre, c'est exister, c'est respirer, c'est se mouvoir, c'est se posséder soimême dans une juste liberté ! La vie d'un peuple, c'est le tempérament qu'il tient de ses pères, l'héritage qu'il en a reçu, l'histoire dont il nourrit son esprit, l'autonomie dont il jouit et qui le protège contre toute force absorbante et tout mélange corrupteur.

Qu'on ne s'y trompe pas : la grandeur, l'importance véritable d'un pays dépend moins du nombre de ses habitants ou de la force de ses armées, que du rayonnement social de ses œuvres et de la libre expansion de sa vie. Qu'était la Grèce dans ses plus beaux jours? un simple lambeau de terre, comme aujourd'hui, tout déchiqueté, pendant aux bords de la Méditerranée, et peuplé à peine de quelques millions [sic] de citoyens. Et cependant qui l'ignore ? de tous les peuples de l'antiquité, nul ne s'est élevé si haut dans l'échelle de la gloire ; 
nul aussi n'a porté si loin l'empire de son génie et n'a marqué d'une plus forte empreinte l'antique civilisation. J'oserai le déclarer : il importe plus à notre race, au prestige de son nom et à la puissance de son action, de garder dans une humble sphère le libre jeu de son organisme et de sa vie que de graviter dans l'orbite de vastes systèmes planétaires.

Du reste, la vie propre ne va guère sans la langue; et l'idiome béni que parlaient nos pères, qui nous a transmis leur foi, leurs exemples, leurs vertus, leurs luttes, leurs espérances, touche de si près à notre mission qu'on ne saurait l'en séparer. La langue d'un peuple est toujours un bien sacré ; mais quand cette langue s'appelle la langue française, quand elle a l'honneur de porter comme dans un écrin le trésor de la pensée humaine enrichi de toutes les traditions des grands siècles catholiques, la mutiler serait un crime, la mépriser, la négliger même, une apostasie. C'est par cet idiome en quelque sorte si chrétien, c'est par cet instrument si bien fait pour répandre dans tous les esprits les clartés du vrai et les splendeurs du beau, pour mettre en lumière tout ce qui ennoblit, tout ce qui éclaire, tout ce qui orne et perfectionne l'humanité, que nous pourrons jouer un rôle de plus en plus utile à l'Église, de plus en plus honorable pour nous-mêmes.

Et ce rôle grandira, croîtra en influence, à mesure que s'élèvera le niveau de notre savoir et que la haute culture intellectuelle prendra chez nous un essor plus ample et plus assuré. Car, on a beau dire, mes Frères, c'est la science qui mène le monde. Cachées sous le voile des sens ou derrière l'épais rideau de la matière, les idées abstraites demeurent, il est vrai, invisibles; mais semblables à cette force motrice que personne ne voit et qui distribue partout avec une si merveilleuse précision la lumière et le mouvement, ce sont elles qui inspirent tous les conseils, qui déterminent toutes les résolutions, qui mettent en branle toutes les énergies. Voilà pour- 
quoi l'importance des universités est si considérable, et pourquoi encore les réjouissances qui auront lieu demain sont si étroitement liées à notre grande fête nationale et en forment, pour ainsi dire, le complément nécessaire ${ }^{29}$.

Ah! l'on me dira sans doute qu'il faut être pratique, que pour soutenir la concurrence des peuples modernes il importe souverainement d'accroître la richesse publique et de concentrer sur ce point tous nos efforts. De fait, tous en conviennent, nous entrons dans une ère de progrès : l'industrie s'éveille ; une vague montante de bien-être, d'activité, de prospérité, envahit nos campagnes ; sur les quais de nos villes, la fortune souriante étage ses greniers d'abondance et le commerce, devenu chaque jour plus hardi, pousse vers nos ports la flotte pacifique de ses navires géants.

À Dieu ne plaise, mes Frères, que je méprise ces bienfaits naturels de la Providence, et que j'aille jusqu'à prêcher à mes concitoyens un renoncement fatal aux intérêts économiques dont ils ont un si vif souci. La richesse n'est interdite à aucun peuple ni à aucune race ; elle est même la récompense d'initiatives fécondes, d'efforts intelligents et de travaux persévérants.

Mais prenons garde ; n'allons pas faire de ce qui n'est qu'un moyen, le but même de notre action sociale. N'allons pas descendre du piédestal où Dieu nous a placés, pour marcher au pas vulgaire des générations assoiffées d'or et de jouissances. Laissons à d'autres nations, moins éprises d'idéal, ce mercantilisme fiévreux et ce grossier naturalisme qui les rivent à la matière. Notre ambition, à nous, doit tendre et viser plus haut; plus hautes doivent être nos pensées, plus hautes nos aspirations. Un publiciste distingué a écrit : «Le matérialisme n'a jamais rien fondé de grand ni de durable ${ }^{30}$. » Cette parole vaut un axiome. Voulons-nous, mes Frères, demeurer fidèles à nous-mêmes, et à la mission supérieure et civilisa- 
trice qui se dégage de toute notre histoire, et qui a fait jusqu'ici l'honneur de notre race ? Usons des biens matériels, non pour eux-mêmes, mais pour les biens plus précieux qu'ils peuvent nous assurer ; usons de la richesse, non pour multiplier les vils plaisirs des sens, mais pour favoriser les plaisirs plus nobles, plus élevés de l'âme ; usons du progrès, non pour nous étioler dans le béotisme qu'engendre trop souvent l'opulence, mais pour donner à nos esprits des ailes plus larges et à nos cœurs un plus vigoureux élan.

Notre vocation l'exige. Et plus nous nous convaincrons de cette vocation elle-même, plus nous en saisirons le caractère vrai et la puissante portée moralisatrice et religieuse, plus aussi nous saurons trouver dans notre patriotisme ce zèle ardent et jaloux, ce courage éclairé et généreux qui, pour faire triompher un principe, ne recule devant aucun sacrifice. L'intelligence de nos destinées nous interdira les molles complaisances, les lâches abandons, les résignations faciles.

Soyons patriotes, mes Frères ; soyons-le en désirs et en paroles sans doute, mais aussi et surtout en action. C'est l'action commune, le groupement des forces, le ralliement des pensées et des volontés autour d'un même drapeau qui gagne les batailles. Et quand faut-il que cette action s'exerce ? quand est-il nécessaire de serrer les rangs ? Ah ! chaque fois que la liberté souffre, que le droit est opprimé, que ce qui est inviolable a subi une atteinte sacrilège ; chaque fois que la nation voit monter à l'horizon quelque nuage menaçant, ou que son cœur saigne de quelque blessure faite à ses sentiments les plus chers.

N'oublions pas non plus que tous les groupes, où circule une même sève nationale, sont solidaires. Il est juste, il est opportun que cette solidarité s'affirme; que tous ceux à qui la Providence a départi le même sang, la même langue, les mêmes croyances, le même souci des choses spirituelles et 
immortelles, resserrent entre eux ces liens sacrés, et poussent l'esprit d'union, de confraternité sociale, aussi loin que le permettent leurs devoirs de loyauté politique. Les sympathies de race sont comme les notions de justice et d'honneur : elles ne connaissent pas de frontières.

Enfin, mes Frères, pour conserver et consolider cette unité morale dont l'absence stériliserait tous nos efforts, rien n'est plus essentiel qu'une soumission filiale aux enseignements de l'Église et une docilité parfaite envers les chefs autorisés qui représentent parmi nous son pouvoir. Cette docilité et cette soumission sont assurément nécessaires à toutes les nations chrétiennes; elles le sont bien davantage à un peuple qui, comme le nôtre, nourri tout d'abord et, pour ainsi dire, bercé sur les genoux de l'Église, n'a vécu que sous son égide, n'a grandi que par ses soins pieux, et poursuit une mission inséparable des progrès de la religion sur ce continent. Plus une société témoigne de respect, plus elle accorde d'estime, de confiance et de déférence au pouvoir religieux, plus aussi elle acquiert de titres à cette protection, parfois secrète, mais toujours efficace, dont Dieu couvre, comme d'un bouclier, les peuples fidèles. Quelle garantie pour notre avenir ! et combien le spectacle de ce jour est propre à affermir notre foi et à soutenir nos meilleures espérances! L'Église et l'État, le clergé et les citoyens, toutes les sociétés, toutes les classes, tous les ordres, toutes les professions, se sont donné la main pour venir au pied de l'autel, en face de Celui qui fait et défait les empires, renouveler l'alliance étroite conclue non loin d'ici, à la naissance même de cette ville, entre la patrie et Dieu. Et pour que rien ne manquât à la solennité de cet acte public, la Providence a voulu qu'un représentant direct de Sa Sainteté Léon XIII ${ }^{31}$, que d'illustres visiteurs ${ }^{32}$, des fils distingués de notre ancienne mère-patrie, rehaussent par leur présence l'éclat et la beauté de cette cérémonie. 
Eh! bien, mes Frères, ce pacte social dont vous êtes les témoins émus, cet engagement national auquel chacun, ce semble, est heureux de souscrire par la pensée et par le cœur, qu'il soit et qu'il demeure à jamais sacré! Qu'il s'attache comme un signe divin au front de notre race! C'est la grande charte qui doit désormais nous régir ${ }^{33}$. Cette charte, où sont inscrits tous les droits, où sont reconnues toutes les saintes libertés, qu'elle soit promulguée partout, sur les portes de nos cités, sur les murs de nos temples, dans l'enceinte de nos parlements et de nos édifices publics! Qu'elle dirige nos législateurs, qu'elle éclaire nos magistrats, qu'elle inspire tous nos écrivains! Qu'elle soit la loi de la famille, la loi de l'école, la loi de l'atelier, la loi de l'hôpital! Qu'elle gouverne, en un mot, la société canadienne tout entière!

De cette sorte, notre nationalité, jeune encore, mais riche des dons du ciel, entrera d'un pas assuré dans la plénitude de sa force et de sa gloire. Pendant qu'autour de nous d'autres peuples imprimeront dans la matière le sceau de leur génie, notre esprit tracera plus haut, dans les lettres et les sciences chrétiennes, son sillon lumineux. Pendant que d'autres races, catholiques elles aussi, s'emploieront à développer la charpente extérieure de l'Église ${ }^{34}$, la nôtre par un travail plus intime et par des soins plus délicats préparera ce qui en est la vie, ce qui en est le cœur, ce qui en est l'âme. Pendant que nos rivaux revendiqueront, sans doute dans des luttes courtoises, l'hégémonie de l'industrie et de la finance, nous, fidèles à notre vocation première, nous ambitionnerons avant tout l'honneur de la doctrine et les palmes de l'apostolat.

Nous maintiendrons sur les hauteurs le drapeau des antiques croyances, de la vérité, de la justice, de cette philosophie qui ne vieillit pas parce qu'elle est éternelle; nous l'élèverons fier et ferme, au-dessus de tous les vents et de tous les orages; nous l'offrirons aux regards de toute l'Amérique 
comme l'emblème glorieux, le symbole, l'idéal vivant de la perfection sociale et de la véritable grandeur des nations.

Alors, mieux encore qu'aujourd'hui, se réalisera cette parole prophétique qu'un écho mystérieux apporte à mes oreilles et qui, malgré la distance des siècles où elle fut prononcée, résume admirablement la signification de cette fête : Eritis mihi in populum, et ego ero vobis in Deum ${ }^{35}$. Vous serez mon peuple, et moi je serai votre Dieu.

Ainsi soit-il, avec la bénédiction de $\mathrm{M}^{\mathrm{gt}} \mathrm{l}^{1}$ Archevêque ${ }^{36}$ !

\section{NOTES}

${ }^{1}$ Ce travail a pu être réalisé grâce à une bourse du CRSH. Nous tenons également à remercier le professeur Jocelyn Létourneau, le Département d'histoire de l'Université Laval et la Fondation de l'Université Laval pour leur soutien, ainsi que les correcteurs de ce texte pour leurs précieux commentaires et suggestions.

${ }^{2}$ Louis-Adolphe Pâquet. Bréviaire du patriote canadien-français. Sermon du 23 juin 1902 commenté par le chanoine Émile Chartier. Montréal, Bibliothèque de l'Action française, 1925.59 p. Ce petit document a été publié et diffusé à des dizaines de milliers d'exemplaire par la Ligue d'Action française. Il va sans dire qu'une telle diffusion ne compte pas pour peu de choses dans la renommée future du texte de Pâquet.

${ }^{3}$ De l'historien Maurice Séguin (1918-1984), maître intellectuel de l'École historique de Montréal, qui fait remonter l'essentiel des problèmes du Canada français aux effets déstructurants de la Conquête de 1760. Le plus bel exemple d'analyse séguiniste du texte de Pâquet se trouve dans l'étude controversée de Michel Brunet, "Trois dominantes de la pensée canadiennefrançaise : l'agriculturisme, l'anti-étatisme et le messianisme». Voir M. Brunet. La présence anglaise et les Canadiens. Montréal, Beauchemin, 1968 [1957], pp. 113-166.

+ La thèse de l'idéalisme, qu'on doit comprendre au sens d'irréalisme, de décalage entre la pensée et la réalité, fut d'abord formulée par Pierre Elliott Trudeau dans son texte «La province de Québec au moment de la grève », qui ouvrait le recueil La grève de l'amiante. Le texte, et les thèses qui y étaient défendues, connurent la fortune que l'on sait (Pierre E. Trudeau, «La province de 
Québec au moment de la grève », dans Trudeau, dir., I a grève de l'amiante, Montréal, Éditions du Jour, 1970 [1956], p. 14). Cette thèse fut reprise et modulée de différentes manières. Jacques Lacoursière, par exemple, se sert du texte comme illustration exemplaire de la dévalorisation de l'activité économique (surtout industrielle) dans le discours des élites nationalistes canadiennes-françaises au tournant du XX $\mathrm{XX}^{\mathrm{e}}$ siècle, et établit implicitement un lien de cause à effet entre cette conception et l'exploitation des ressources naturelles du Québec par des intérêts étrangers, notamment américains (J. Lacoursière, Histoire populaire du Québec, tome IV, 1896 à 1960, Sillery, Septentrion, 1997, pp. 35-36). Robert Sargent, quoiqu'un peu plus nuancé, n'en arrive pas moins à une conclusion similaire (R. Sargent, The Thought of Monseigneur LouisAdolphe Pâquet as a Spokesman for French Canadian Ultramontanism, Thèse de Th.D., Union Theological Seminary, New York, 1968, pp. 327-328). Par ailleurs, deux autres critiques, qui inscrivent leur analyse dans un cadre plus ou moins marxiste, se montrent d'une dureté sans concession a l'endroit du texte de Pâquet. Denis Monière, d'une part, fait de l'extrait du discours que nous citons en introduction l'expression à la fois claire, synthétique et exemplaire d'un projet de société « directement opposé aux tendances nouvelles du monde matériel », par conséquent « irréaliste » et «attardé », mis de l'avant par une élite "rétrograde » (D. Monière, Le développement des idéologies au Québec des origines à nos jours, Montréal, Québec-Amérique, 1977, pp. 227 et 260 ; Pour comprendre le nationalisme au Québec et ailleurs, Montréal, Presses de l'Université de Montréal, 2001, pp. 96-97). Georges Vincenthier, pour sa part, trouve dans ce texte l'illustration d'une pensée « idéaliste », « obstinément conservatrice, intégriste et triomphante " qui reflète la "vacuité intellectuelle ", l'« autoglorification impertinente » et la « grandiloquence impuissante » qui seraient caractéristiques de la vie de l'esprit au Canada français à la fin du XIX siècle (G. Vincenthier, Histoire des idées au Québec, des troubles de 1837 au référendum de 1980, Montréal, VLB Éditeur, 1983, p. 80). Ces exemples montrent quelques-unes des figures qu'ont prises les différentes critiques du texte de Pâquet. Il faut noter deux exceptions à cette tendance générale, deux analyses qui ont tenté de nuancer les interprétations courantes du dit texte. D'abord, William F. Ryan passe en revue les différentes interprétations possibles du texte, en prenant bien soin cependant d'adopter une perspective d'ensemble sur la réflexion de l'auteur concernant le Canada français et la vie économique. Il nous offre ainsi un bilan court mais nuancé (W.F. Ryan, The Clergy and Economic Growth in Quebec (1896-1914), Québec, Presses de l'Université Laval, 1968, pp. 248-249. Cet ouvrage, notons-le, mériterait d'être traduit.). Fernande Roy, quant à elle, fait rapidement mention du texte de Pâquet dans sa petite introduction à l'histoire des idéologies au Québec, mais tente tout de même 
de lui rendre justice dans ce cadre forcément très limité, ce qu'elle réussit assez bien. Elle en profite même pour lancer une petite pointe aux analystes qui rendent compte de l'argumentation de façon trop étroitement sélective (F. Roy, Histoire des idéologies an Québec aux XIX' et XX' siècles, Montréal, Boréal, 1993, p. 66). Le lecteur intéressé à embrasser d'une manière plus générale la pensée et l'œuvre de $\mathrm{M}^{\mathrm{gt}}$ Pâquet pourra consulter la petite anthologie préparée par Yvan Lamonde, Louis-Adolphe Paquet. Montréal, Fides, 1972. 86 p. (Collection «Classiques canadiens »).

${ }^{5}$ Québec, Demers et Frères, 1893-1903, 6 volumes.

'Québec, 1908-1915, 4 volumes.

${ }^{7}$ Québec, Imprimerie franciscaine missionnaire, 1917-1934, 7 volumes.

${ }^{8}$ Sargent, op. cit., p. v.

" [S.a.], « Pâquet, Louis-Adolphe », dans Réginal Hamel, John Hare et Paul Wyczynski, dir., Dictionnaire des auteurs de langue française en Amérique du Nord, Montréal, Fides, 1989, pp. 1058-1059.

11) Yvan Lamonde, «Un almanach idéologique des années 1900-1929 : l'œuvre de Monseigneur Louis-Adolphe Pâquet, théologien nationaliste ", dans Fernand Dumont et al., (dir.), Idéologies au Canada français 1900-1929, Québec, Presses de l'Université Laval, 1974, pp. 253-254.

11 Le Soleil, 24 juin 1902, pp. 1 et 3.

${ }^{12}$ Edmund Burke, Reflections on the Revolution in France, Stanford, Stanford University Press, 2001, pp. 260-261.

${ }^{13}$ Lamonde, loc. cit., p. 264.

${ }^{14}$ La version du texte présentée ici est une retranscription de l'édition du chanoine Chartier, Bréviaire du patriote canadien-français, op. cit. pp. 49-59.

${ }^{15}$ Jean Dolbeau, récollet.

${ }^{16}$ On notera que chaque expression latine qui se trouve dans le texte est immédiatement traduite par Pâquet.

${ }^{17}$ L'interprétation que donne Émile Chartier de ce passage est intéressante. "Le "front" désigne ici les autorités de France, de la pensée de qui nous sommes issus. Le "cœur" résume les sentiments de ceux qui furent poussés sur nos bords par le souffle de cette pensée. Les "lèvres" représentent les enseignements que propagèrent, parmi les infidèles de ce pays, les hommes 
animés des sentiments pieux et des préoccupations civilisatrices de la France. » (Chartier, dans Pâquet, Bréviaire..., p. 14.)

${ }^{18}$ Il s'agit de l'encyclique Nobilissima gallorum gens, promulguée par le pape Léon XIII en 1884.

${ }^{19} \mathrm{La}$ France révolutionnaire : moderne, rationaliste et libérale.

${ }^{20}$ Référence, d'une part, aux souffrances et à la persécution dont furent l'objet les catholiques de France à la suite de la Révolution, et, d'autre part, à l'exil de centaines de membres des congrégations religieuses françaises à la suite de l'adoption de la loi du $1^{\text {er }}$ juillet 1901 sur les associations, dirigée contre les congrégations enseignantes, qui prévoyait notamment la fermeture des écoles congréganistes non autorisées. Par ailleurs, les « treize siècles de foi généreuse et de dévouement sans égal à la cause du droit chrétien » remontent selon toute vraisemblance au baptême de Clovis ${ }^{\text {Ier }}$, roi des Francs, en 498 ou 499.

${ }^{21}$ Les personnages dont Pâquet fait mention comptent parmi les plus illustres membres d'une sorte de panthéon à la gloire de la France catholique : Clovis I $^{\text {er }}$ (vers 466-511), roi des Francs, et Charlemagne (742-814), roi des Francs et sacré " empereur d'Occident ", tous deux artisans de la première heure de la grandeur du christianisme et de ce qui allait devenir la France ; Louis IX, dit saint Louis (1214-1270), roi de France, croisé, grand chrétien, sous le règne duquel la France connut un grand rayonnement intellectuel et artistique (notamment grâce à la fondation de la Sorbonne) ; Jeanne d'Arc (1412-1431), héroïne inspiratrice de l'armée française à la fin de la Guerre de Cent Ans, dont le culte connut un renouveau à la suite de la guerre francoallemande de 1870-1871 et est toujours célébré de nos jours par les héritiers spirituels de la France catholique ; saint Vincent de Paul (1576-1660), prêtre français qui se distingua par ses grandes œuvres charitables, et Bossuet (16271704), prêtre, théologien et homme de lettres de renom, tous deux puissants symboles de la Contre-Réforme.

${ }^{22}$ Ferland, Cours d'bistoire du Canada, vol. 1, p. 31. [Note de Pâquet]

${ }^{23}$ Casgrain, Histoire de l'Hôtel-Dieu de Québec, p. 73. [Note de Pâquet]

${ }^{24}$ Référence aux missionnaires jésuites qui ont été tués et dans certains cas suppliciés par les Iroquois lors de la destruction de la Huronie à la fin des années 1640, les «Saints Martyrs canadiens » : René Goupil, Isaac Jogues, Jean de la Lande, Antoine Daniel, Jean de Brébeuf, Gabriel Lalemant, Charles Garnier et Noël Chabanel. Ils furent tous canonisés en 1930 par Pie XI.

${ }^{25}$ Bâton que Dieu commanda à Moïse de prendre pour opérer ses miracles. 
${ }^{26}$ À l'époque, l'Université Laval comptait deux campus : le campus principal de Québec et une succursale à Montréal, fondée en 1876 et ouverte depuis 1878. En 1920, la succursale montréalaise gagna sa pleine indépendance et devint l'Université de Montréal.

${ }^{27}$ Pâquet fait ici référence à l'importante population de souche française installée hors du Québec. Vers 1900, on comptait environ 1320000 Canadiens français au Québec pour environ 900000 francophones dispersés dans les autres provinces canadiennes et aux États-Unis, soit environ $330000 \mathrm{au} \mathrm{Ca-}$ nada, et 570000 aux États-Unis. (Paul-André Linteau, René Durocher et Jean-Claude Robert, Histoire du Québec contemporain, tome 1, De la Confédération à la crise (1867-1929), Montréal, Boréal, 1989, pp. 48-49; Yves Roby, Les Franco-Américains de la Nouvelle-Angleterre. Rêves et réalités, Québec, Septentrion, 2000, p. 11.)

${ }^{28}$ Es 43.21.

${ }^{29}$ Le lendemain, 24 juin 1902, on célébrait en grande pompe le cinquantenaire de l'Université Laval.

${ }^{30}$ Rameau de Saint-Père, La France aux colonies. [Note de Pâquet]

${ }^{31} \mathrm{M}^{\mathrm{gr}}$ Falconio, délégué apostolique au Canada.

${ }^{32}$ De toute évidence, Pâquet fait ici surtout référence à Raymond Thamin, recteur de l'Université de Rennes et délégué des universités françaises aux fêtes du cinquantenaire de l'Université Laval (Camille Roy, L'Université Laval et les fêtes du cinquantenaire, Québec, Typ. Dussault et Proulx, 1903, p. 318 et suiv.).

${ }^{33}$ Il faut noter ici la convergence de « alliance... entre la patrie et Dieu » (paragraphe précédent), «pacte social», « engagement national» et " grande charte».

${ }^{34}$ Sans doute une référence aux Irlandais...

${ }^{35} \mathrm{Jr} 11.4$.

${ }^{36}$ M ${ }^{g r}$ Louis-Nazaire Bégin (1840-1925), archevêque de Québec de 1898 à 1925, promu au cardinalat en $1914 . \mathrm{M}^{\mathrm{gr}}$ Bégin était le célébrant de messe. 